\title{
PARAMETER-UNIFORM FITTED MESH METHOD FOR QUASILINEAR DIFFERENTIAL EQUATIONS WITH BOUNDARY LAYERS ${ }^{1}$
}

\author{
PAUL A. FARRELL \\ Kent State University \\ Kent, Ohio 44242, U.S.A. \\ E-mail: farrell@mcs.dune.edu \\ EUGENE O'RIORDAN \\ Dublin City University \\ Glasnevin, Dublin 9, Ireland \\ E-mail: Eugene.ORiordan@dcu.ie \\ JOHN J.H. MILLER \\ Trinity College \\ Dublin 2, Ireland \\ E-mail: jmiller@tcd.ie
}

\section{GRIGORII I. SHISHKIN}

Institute of Mathematics and Mechanics, Ural Branch of the Russian Academy of Sciences 16 S. Kovalevskaya Str., 620219 Ekaterinburg, Russia

E-mail: Grigorii@shishkin.ural.ru

\begin{abstract}
Singularly perturbed quasilinear boundary value problems exhibiting boundary layers are considered. Special piecewise-uniform meshes are constructed which are fitted to these boundary layers. Numerical methods composed of upwind difference operators and these fitted meshes are shown to be parameter robust, in the sense that the solutions satisfy an error estimate in the maximum norm which is independent of the value of the singular perturbation parameter. Numerical results supporting the theory are presented.
\end{abstract}

2000 Mathematics Subject Classification: 34E10; 65L10.

Keywords: quasilinear boundary value problem, singular perturbation, finitedifference method, piecewise uniform mesh, $\varepsilon$-uniform error estimate.

\section{Introduction}

Differential equations with a small parameter $\varepsilon$ multiplying the highest order derivative terms are said to be singularly perturbed and normally boundary layers occur in their solutions.

\footnotetext{
${ }^{1}$ The work was supported in part by the Enterprise Ireland grant SC-98-612 and by the Russian Foundation for Basic Research under Grant N 01-01-01022.
} 
Singularly perturbed differential equations are ubiquitous in mathematical problems in the sciences and engineering. For example, the Navier-Stokes equations of fluid flow at high Reynolds number, the equations governing flow in porous media, the drift-diffusion equations of semiconductor device physics [11,18], and mathematical models of liquid crystal materials and of chemical reactions [24].

Classical numerical methods usually give unsatisfactory numerical results when the singular perturbation parameter $\varepsilon$ is small. In particular, the pointwise errors in numerical methods based on centered or upwinded differences on uniform meshes depend inversely on a power of $\varepsilon$. In this paper we construct numerical methods for solving singularly perturbed quasilinear differential equations whose convergence behavior in the global maximum norm is parameter-uniform, that is $\varepsilon$-uniform since the singular perturbation parameter is $\varepsilon$.

It is of both theoretical and practical interest to construct parameter-uniform numerical methods of guaranteed accuracy for such problems. These are $\varepsilon$-uniform, in the sense that there exists an $N_{0}$, independent of $\varepsilon$, such that for all $N \geqslant N_{0}$, where $N$ is the number of mesh elements, the error constant $C$ and order of convergence $p$ in the global maximum norm are independent of $\varepsilon$. Parameter-uniform behavior may be achieved by fitting the mesh or by fitting the finite difference operator to the boundary layer. Such methods are called parameter-uniform fitted mesh [5] or fitted operator methods [17].

Parameter-uniform methods consisting of exponentially fitted finite difference operators on uniform meshes are thoroughly investigated and applied successfully to singularly perturbed boundary value problems for linear ordinary differential equations in $[1,17]$ and to linear partial differential equations in $[7,19,20]$. In the case of nonlinear problems, $\varepsilon$-uniform convergence in the $\ell_{1}$ norm on a uniform mesh is proved for semilinear problems in $[15,23]$ and for quasilinear problems in [14]. In [3] it was proved that for semilinear problems $\varepsilon$ uniform convergence in the maximum norm is not achievable using fitted schemes with frozen coefficients on uniform meshes. Note that an $\varepsilon$-uniform numerical method for such semilinear problems using a standard finite difference operator on a fitted piecewise-uniform mesh is given in [4].

Linß et al. $[6,8]$ examine numerical methods based on Shishkin-type fitted meshes for quasilinear convection-diffusion problems of the type considered in this paper. They prove convergence results in the discrete $L_{\infty}$-norm, under the restriction of $\varepsilon \leqslant C N^{-1}$ and homogeneous boundary conditions. Their proofs are based on discrete Green's function arguments, which are difficult to extend to partial differential equations. In this paper, the proof relies on barrier function and comparison principle arguments.

Shishkin $[21,22]$ was the first to provide a complete $\varepsilon$-uniform $L_{\infty}$ analysis for a singularly perturbed quasilinear problem in more than one dimension. The order of convergence for this general class of partial differential equations was proven to be less than 0.5 . In the present paper, we show that the order is almost one when applied to the subclass of ordinary differential equations. The proof presented here follows the same argument as that outlined in $[21,22]$ for partial differential equations. The proof presented here is not restricted to the case of $\varepsilon \leqslant C N^{-1}$ and is extended to a global convergence result. In contrast to the papers of Shishkin, we present numerical results based on extensive numerical experiments, we discuss the nonlinear solver and the dependence on initial guesses. This paper examines these effects in the case of one-dimensional problems.

In Section 2, the continuous quasilinear problems are formulated and bounds on the derivatives of their exact solutions are derived. In Section 3, a fitted mesh finite difference method is constructed and proved theoretically to be $\varepsilon$-uniform. Then, in Section 4, nu- 
merical results are presented which validate computationally the theoretical results. The technical details on how to construct a suitable barrier function are given in an Appendix.

\section{Analytical results}

Consider the class of quasilinear Dirichlet problems

$$
\begin{gathered}
\varepsilon u_{\varepsilon}^{\prime \prime}+b\left(u_{\varepsilon}\right) u_{\varepsilon}^{\prime}=f, \quad x \in \Omega=(0,1), \\
u_{\varepsilon}(0)=A, \quad u_{\varepsilon}(1)=B, \\
\beta \geqslant b\left(u_{\varepsilon}\right)>\alpha>0, \quad\left(x, u_{\varepsilon}\right) \in \bar{\Omega} \times \Re, \\
\left\|\frac{\partial b}{\partial u_{\varepsilon}}\right\| \leqslant M,
\end{gathered}
$$

where $\beta, \alpha$ are constants independent of $\varepsilon$ and $b(\cdot), f$ are sufficiently smooth. The existence and uniqueness of $u_{\varepsilon}$ follow from the arguments given in [10]. A further restriction (see (3.4) in Section 3) is placed on the class in order to derive our main theoretical result in Theorem 4. Note that $b\left(u_{\varepsilon}\right)$ is strictly bounded away from zero, and so the interesting interior layer phenomena arising in Burgers' equation are excluded from consideration in the present paper.

The parameter $\varepsilon$ takes arbitrary values in the half-open interval $(0,1]$, and $\|\cdot\|$ denotes the global maximum norm taken over the appropriate domain of the independent variable. That is,

$$
\|f\|=\max _{x \in \bar{\Omega}}|f(x)| .
$$

Throughout this paper, $C$ and $M$ denote generic positive constants that are independent of $\varepsilon$ and, in the case of discrete problems, are also independent of the mesh parameter $N$. A function $u_{\varepsilon} \in C^{2}(\Omega) \cap C^{0}(\bar{\Omega})$ is said to be a solution of the boundary value problem (2.1) if it satisfies (2.1a) on $\Omega$ and (2.1b) on $\partial \Omega=\bar{\Omega} \backslash \Omega$.

In this section a number of results from the theory of differential equations are stated and proved. These give the required bounds on the derivatives of the exact solution of problem (2.1). In accord with (2.1) it is useful to introduce the notation

$$
L_{u}(v(x)) \equiv \varepsilon v^{\prime \prime}+b\left(u_{\varepsilon}\right) v^{\prime} \quad \text { for any } v \in C^{2}(\Omega) .
$$

Note that $L_{u}\left(v_{1}+v_{2}\right)=L_{u}\left(v_{1}\right)+L_{u}\left(v_{2}\right)$. The following comparison principle holds

Lemma 2.1. Let $w_{i} \in C^{2}(\Omega) \cap C^{0}(\bar{\Omega})$ for $i=1,2$ and $x \in \bar{\Omega}$. Suppose that for all $x \in \Omega$, the inequalities:

$$
L_{u}\left(w_{1}(x)\right) \geqslant L_{u}\left(u_{\varepsilon}(x)\right) \geqslant L_{u}\left(w_{2}(x)\right)
$$

hold, whenever $w_{1}(x) \leqslant u_{\varepsilon}(x) \leqslant w_{2}(x)$ for all $x \in \partial \Omega$. Then, for all $x \in \bar{\Omega}$, the function $u_{\varepsilon}$ satisfies the following inequalities

$$
w_{1}(x) \leqslant u_{\varepsilon}(x) \leqslant w_{2}(x) .
$$

Proof. Follow the same argument as for the linear operator $L u=\varepsilon u^{\prime \prime}+b(x) u^{\prime}$. The proof is by contradiction. Assume that there exists a point $p \in \bar{\Omega}$ where $w_{2}(p)-u_{\varepsilon}(p)<0$. It follows from the hypotheses that $p \notin \partial \Omega$. Define the auxiliary function $v=e^{\alpha x /(2 \varepsilon)}\left(\left(w_{2}-u_{\varepsilon}\right)(x)\right)$ 
and note that $v(p)<0$. Choose $q \in \Omega$ such that $v(q)=\min _{\Omega} v(x)<0$. Therefore, from the definition of $q$ we have $v^{\prime}(q)=0$ and $v^{\prime \prime}(q) \geqslant 0$. But then

$$
L_{u}\left(w_{2}-u_{\varepsilon}\right)(q)=\left(\varepsilon v^{\prime \prime}(q)+\left(b\left(u_{\varepsilon}\right)-\alpha\right) v^{\prime}(q)-\frac{\alpha}{2 \varepsilon}\left(b\left(u_{\varepsilon}\right)-\alpha / 2\right) v(q)\right) e^{-q \alpha /(2 \varepsilon)}>0
$$

which is a contradiction. An analogous argument applies to $w_{1}(x)-u_{\varepsilon}(x)$.

From this lemma it follows that

$$
\min \{A, B\}-\frac{\left\|L_{u} u_{\varepsilon}\right\|}{\alpha}(1-x) \leqslant u_{\varepsilon}(x) \leqslant \max \{A, B\}+\frac{\left\|L_{u} u_{\varepsilon}\right\|}{\alpha}(1-x) .
$$

The following theorem gives bounds on the derivatives of the exact solution $u_{\varepsilon}$ of problem (2.1).

Theorem 2.1. Let $u_{\varepsilon}$ be the solution of (2.1). Then for $k=1,2,3$

$$
\left\|u_{\varepsilon}^{(k)}\right\|_{\Omega} \leqslant C \varepsilon^{-k}
$$

Proof. By the Mean Value Theorem, there exists a point $z \in(0, \varepsilon)$ such that

$$
u_{\varepsilon}^{\prime}(z)=\frac{u_{\varepsilon}(\varepsilon)-u_{\varepsilon}(0)}{\varepsilon}
$$

and so $\left|\varepsilon u_{\varepsilon}^{\prime}(z)\right| \leqslant 2\left\|u_{\varepsilon}\right\|$. Integrating the differential equation, we get

$$
\varepsilon u_{\varepsilon}^{\prime}(z)-\varepsilon u_{\varepsilon}^{\prime}(0)=\int_{0}^{z} f(t) d t-\int_{u_{\varepsilon}(0)}^{u_{\varepsilon}(z)} b(t) d t .
$$

It follows that $\left|\varepsilon u_{\varepsilon}^{\prime}(0)\right| \leqslant\|f\|+C\left\|u_{\varepsilon}\right\|$. Using (2.4) with $z=x$, we see that $\left|\varepsilon u_{\varepsilon}^{\prime}(x)\right| \leqslant$ $2\|f\|+C\left\|u_{\varepsilon}\right\|$ for all $x \in \Omega$, and the required result for $k=1$ follows. Now from the differential equation we have

$$
\varepsilon u_{\varepsilon}^{\prime \prime}=f-b\left(u_{\varepsilon}\right) u_{\varepsilon}^{\prime} \quad \text { and } \quad \varepsilon u_{\varepsilon}^{\prime \prime \prime}=\left(f-b\left(u_{\varepsilon}\right) u_{\varepsilon}^{\prime}\right)^{\prime}=f^{\prime}-b\left(u_{\varepsilon}\right) u_{\varepsilon}^{\prime \prime}-b_{u}\left(u_{\varepsilon}\right)\left(u_{\varepsilon}^{\prime}\right)^{2}
$$

from which we obtain successively the required bounds on the second and third derivatives.

In order to prove that the numerical method is $\varepsilon$-uniform, more precise information on the behavior of the exact solution of problem (2.1) is needed. This is obtained by writing the solution in the form

$$
u_{\varepsilon}(x)=v_{\varepsilon}(x)+w_{\varepsilon}(x), \quad x \in \bar{\Omega},
$$

where $v_{\varepsilon}$ and $w_{\varepsilon}$ are, respectively, the regular and singular components of $u_{\varepsilon}$. The regular component $v_{\varepsilon}$ is the solution of

$$
L_{v}\left(v_{\varepsilon}(x)\right)=f(x) \text { in } \Omega, \quad v_{\varepsilon}(1)=u_{\varepsilon}(1) \quad \text { and } \quad v_{\varepsilon}(0)=v_{0}(0)+\varepsilon v_{1}(0)
$$

where $v_{0}, v_{1}$ are defined by

$$
\begin{aligned}
& b\left(v_{0}\right)\left(v_{0}(x)\right)^{\prime}=f(x), \quad x \in \Omega, \quad v_{0}(1)=u_{\varepsilon}(1), \\
& \varepsilon v_{0}^{\prime \prime}(x)+b\left(v_{0}+\varepsilon v_{1}\right)\left(v_{0}(x)+\varepsilon v_{1}(x)\right)^{\prime}=f(x), \quad x \in \Omega, \quad v_{1}(1)=0 \text {. }
\end{aligned}
$$


Finally, since $v_{\varepsilon}$ is defined and $u_{\varepsilon}=v_{\varepsilon}+w_{\varepsilon}$, the singular component $w_{\varepsilon}$ is determined by

$$
L_{u}\left(u_{\varepsilon}(x)\right)=f(x) \text { in } \Omega, \quad u_{\varepsilon}(0)=A, \quad u_{\varepsilon}(1)=B .
$$

Separate estimates for the derivatives of $v_{\varepsilon}$ and $w_{\varepsilon}$ with explicit $\varepsilon$-dependence are given in the following theorem.

Theorem 2.2. Let $u_{\varepsilon}$ be the solution of (2.1) and let $u_{\varepsilon}=v_{\varepsilon}+w_{\varepsilon}$. For all $k \leqslant 3$ and for sufficiently small $\varepsilon$, the derivatives of $v_{\varepsilon}$ and $w_{\varepsilon}$ satisfy the following bounds:

$$
\begin{aligned}
& \left\|v_{\varepsilon}^{(k)}\right\|_{\bar{\Omega}} \leqslant C \varepsilon^{2-k}, \\
& \left|w_{\varepsilon}(x)\right| \leqslant C \exp \left(-\alpha \varepsilon^{-1} x\right), \quad x \in \bar{\Omega} .
\end{aligned}
$$

Also

$$
\left\|w_{\varepsilon}^{(k)}\right\| \leqslant C \varepsilon^{-k} \exp \left(-\alpha \varepsilon^{-1} x\right), \quad x \in \bar{\Omega} .
$$

Proof. Note that $v_{0}$ and its derivatives are independent of $\varepsilon$ and

$$
v e v_{0}^{\prime \prime}+b\left(v_{0}+\varepsilon v_{1}\right)\left(v_{0}+\varepsilon v_{1}\right)^{\prime}=f(x)=b\left(v_{0}\right)\left(v_{0}\right)^{\prime}, \quad v_{1}(1)=0 .
$$

Hence

$$
\varepsilon v_{0}^{\prime \prime}+\varepsilon b\left(v_{0}+\varepsilon v_{1}\right) v_{1}^{\prime}+\left(b\left(v_{0}+\varepsilon v_{1}\right)-b\left(v_{0}\right)\right) v_{0}^{\prime}=0 .
$$

For all $x \in(0,1)$, there exists $\hat{v}(x)$ such that

$$
b\left(v_{0}+\varepsilon v_{1}\right)-b\left(v_{0}\right)=\varepsilon b^{\prime}(\hat{v}) v_{1} .
$$

So the function $v_{1}$ satisfies the initial value problem

$$
b\left(v_{0}+\varepsilon v_{1}\right) v_{1}^{\prime}+\left(b^{\prime}(\hat{v}) v_{0}^{\prime}\right) v_{1}=-v_{0}^{\prime \prime}, \quad v_{1}(1)=0 .
$$

Hence $\left|v_{1}\right| \leqslant C$ and $\left|v_{1}^{\prime}\right| \leqslant C$. Differentiate the equation defining $v_{1}$ to bound $\left|v_{1}^{\prime \prime}\right|$ and $\left|v_{1}^{\prime \prime \prime}\right|$. We can write $v_{\varepsilon}=v_{0}+\varepsilon v_{1}+\varepsilon^{2} v_{2}$, where $v_{2}$ is the solution of

$$
\varepsilon\left(v_{0}^{\prime \prime}+\varepsilon v_{1}^{\prime \prime}+\varepsilon^{2} v_{2}^{\prime \prime}\right)+b\left(v_{\varepsilon}\right)\left(v_{0}^{\prime}+\varepsilon v_{1}^{\prime}+\varepsilon^{2} v_{2}^{\prime}\right)=f=\varepsilon v_{0}^{\prime \prime}+b\left(v_{0}+\varepsilon v_{1}\right)\left(v_{0}+\varepsilon v_{1}\right)^{\prime} .
$$

Hence

$$
\varepsilon v_{2}^{\prime \prime}+b\left(v_{\varepsilon}\right) v_{2}^{\prime}+\varepsilon^{-2}\left(b\left(v_{\varepsilon}\right)-b\left(v_{0}+\varepsilon v_{1}\right)\right)\left(v_{0}+\varepsilon v_{1}\right)^{\prime}=-v_{1}^{\prime \prime}
$$

which can be written in the form

$$
\varepsilon v_{2}^{\prime \prime}+b\left(v_{\varepsilon}\right) v_{2}^{\prime}+\left(b^{\prime}(\tilde{v})\left(v_{0}+\varepsilon v_{1}\right)^{\prime}\right) v_{2}=-v_{1}^{\prime \prime}, \quad v_{2}(0)=v_{2}(1)=0 .
$$

Hence, from the previous theorem, $\left\|v_{2}^{(k)}\right\|_{\bar{\Omega}} \leqslant C \varepsilon^{-k}$. Now we estimate the singular term. Note that

$$
\varepsilon u_{\varepsilon}^{\prime \prime}+b\left(u_{\varepsilon}\right) u_{\varepsilon}^{\prime}=f=\varepsilon v_{\varepsilon}^{\prime \prime}+b\left(v_{\varepsilon}\right) v_{\varepsilon}^{\prime}
$$

and so

$$
\varepsilon w_{\varepsilon}^{\prime \prime}+\left(b\left(u_{\varepsilon}\right)-b\left(v_{\varepsilon}\right)\right) v_{\varepsilon}^{\prime}+b\left(u_{\varepsilon}\right) w_{\varepsilon}^{\prime}=\varepsilon w_{\varepsilon}^{\prime \prime}+b\left(u_{\varepsilon}\right) w_{\varepsilon}^{\prime}+\left(b^{\prime}(\hat{w}) v_{\varepsilon}^{\prime}\right) w_{\varepsilon}=0
$$

and $w_{\varepsilon}(0)=u_{\varepsilon}(0)-v_{\varepsilon}(0), w_{\varepsilon}(1)=0$. Choose $\varepsilon$ sufficiently small so that

$$
b^{2}\left(u_{\varepsilon}\right)-4 \varepsilon b^{\prime}(\hat{w}) v_{\varepsilon}^{\prime}>0 .
$$

Then we can apply the arguments from the linear problem [5] to get the bounds on $w_{\varepsilon}$ and its derivatives. 


\section{Nonlinear finite difference methods}

In this section finite difference methods with piecewise-uniform fitted meshes are constructed for problem (2.1). The fitted piecewise-uniform mesh $\bar{\Omega}_{\varepsilon}^{N}$ on the interval $[0,1]$ is formed by partitioning the interval into two subintervals $[0, \sigma],[\sigma, 1]$, where $\sigma \equiv \min [0.5, C \varepsilon \ln N]$ and $C>1 / \alpha$. On each of these subintervals, a uniform mesh is constructed. A monotone finite difference method for problem $(2.1)$, on the mesh $\bar{\Omega}_{\varepsilon}^{N}$ is now defined by

$$
\begin{aligned}
L_{U_{\varepsilon}}^{N}\left(U_{\varepsilon}(x)\right) & =f(x), \quad x \in \Omega_{\varepsilon}^{N}, \\
U_{\varepsilon}(0) & =A, \quad U_{\varepsilon}(1)=B,
\end{aligned}
$$

where $L_{Z}^{N}(Z(x)) \equiv\left\{\varepsilon \delta^{2}+b(Z) D^{+}\right\} Z(x)$ and

$$
\begin{gathered}
D^{+} Z\left(x_{i}\right)=\left(Z\left(x_{i+1}\right)-Z\left(x_{i}\right)\right) / h_{i+1}, \quad D^{-} Z\left(x_{i}\right)=\left(Z\left(x_{i}\right)-Z\left(x_{i-1}\right)\right) / h_{i}, \\
\delta^{2} Z\left(x_{i}\right)=2\left(D^{+}-D^{-}\right) Z\left(x_{i}\right) /\left(h_{i}+h_{i+1}\right), \quad h_{i}=x_{i}-x_{i-1} .
\end{gathered}
$$

The existence and uniqueness of $U_{\varepsilon}$ again follow from the arguments given in [10]. The solution of the nonlinear difference scheme is decomposed into $U_{\varepsilon}=V_{\varepsilon}+W_{\varepsilon}$ where $V_{\varepsilon}$ is the solution

$$
\begin{gathered}
\left\{\varepsilon \delta^{2}+b\left(V_{\varepsilon}\right) D^{+}\right\} V_{\varepsilon}=f, \quad x \in \Omega_{\varepsilon}^{N}, \\
V_{\varepsilon}(0)=v_{\varepsilon}(0), \quad V_{\varepsilon}(1)=v_{\varepsilon}(1) .
\end{gathered}
$$

The error is written in the form

$$
U_{\varepsilon}-u_{\varepsilon}=V_{\varepsilon}-v_{\varepsilon}+W_{\varepsilon}-w_{\varepsilon}
$$

and these error components $V_{\varepsilon}-v_{\varepsilon}$ and $W_{\varepsilon}-w_{\varepsilon}$ are bounded separately.

Theorem 3.1. The smooth component of the error satisfies the following E-uniform error estimate:

$$
\sup _{0<\varepsilon \leqslant \varepsilon_{0}}\left\|V_{\varepsilon}-v_{\varepsilon}\right\|_{\bar{\Omega}_{\varepsilon}^{N}} \leqslant C N^{-1}
$$

where $\varepsilon_{0}$ is sufficiently small and $C$ is a constant independent of $\varepsilon$ and $N$.

Proof.

$$
\begin{aligned}
\left\{\varepsilon \delta^{2}+b\left(V_{\varepsilon}\right) D^{+}\right\}\left(V_{\varepsilon}-v_{\varepsilon}\right) & =\varepsilon v_{\varepsilon}^{\prime \prime}+b\left(v_{\varepsilon}\right) v_{\varepsilon}^{\prime}-\left\{\varepsilon \delta^{2}+b\left(V_{\varepsilon}\right) D^{+}\right\}\left(v_{\varepsilon}\right) \\
& =\varepsilon\left(v_{\varepsilon}^{\prime \prime}-\delta^{2} v_{\varepsilon}\right)-b\left(V_{\varepsilon}\right) D^{+} v_{\varepsilon}+b\left(v_{\varepsilon}\right) v_{\varepsilon}^{\prime} \\
& =\varepsilon\left(v_{\varepsilon}^{\prime \prime}-\delta^{2} v_{\varepsilon}\right)+b\left(V_{\varepsilon}\right)\left(v_{\varepsilon}^{\prime}-D^{+} v_{\varepsilon}\right)+\left(b\left(v_{\varepsilon}\right)-b\left(V_{\varepsilon}\right)\right) v_{\varepsilon}^{\prime} \\
& =\varepsilon\left(v_{\varepsilon}^{\prime \prime}-\delta^{2} v_{\varepsilon}\right)+b\left(V_{\varepsilon}\right)\left(v_{\varepsilon}^{\prime}-D^{+} v_{\varepsilon}\right)+b^{\prime}\left(\zeta_{i}\right)\left(v_{\varepsilon}-V_{\varepsilon}\right) v_{\varepsilon}^{\prime}
\end{aligned}
$$

Introduce the linear difference operator

$$
M_{V}^{N} Z \equiv\left(\varepsilon \delta^{2}+b\left(V_{\varepsilon}\right) D^{+}+\left(b^{\prime}\left(\zeta_{i}\right)\right) w_{\varepsilon}^{\prime}\right) Z,
$$

where $\zeta_{i}$ is defined implicitly by

$$
b_{u}\left(\zeta_{i}\right)\left(V_{\varepsilon}-v_{\varepsilon}\right) \equiv b\left(V_{\varepsilon}\right)-b\left(v_{\varepsilon}\right) .
$$


This difference operator satisfies a discrete maximum principle, provided that the following inequality holds for all $x$ :

$$
b^{2}\left(V_{\varepsilon}\right)-4 \varepsilon_{0}\left(b_{u}\left(\zeta_{i}\right) v_{\varepsilon}^{\prime}\right)>0 .
$$

Using the bounds in Theorem 2 and standard local truncation error estimates, we get

$$
\left|M_{V}^{N}\left(V_{\varepsilon}-v_{\varepsilon}\right)\left(x_{i}\right)\right| \leqslant C N^{-1} .
$$

With the two functions $\psi^{ \pm}\left(x_{i}\right)=C N^{-1}\left(1-x_{i}\right) \pm\left(V_{\varepsilon}-v_{\varepsilon}\right)\left(x_{i}\right)$, and the discrete maximum principle the proof is completed in the usual way.

Note that (3.3) is a sufficient, but not a necessary condition on $\varepsilon_{0}$.

Lemma 3.1. For the smooth component $V_{\varepsilon}$ of the solution of (3.1) the following $\varepsilon$ uniform bounds

$$
\left|D^{+} V_{\varepsilon}\left(x_{i}\right)\right| \leqslant C, \quad \quad x_{i} \geqslant \sigma
$$

and

$$
\left|D^{+} V_{\varepsilon}\left(x_{i}\right)\right| \leqslant C\left(1+\frac{N^{-1}}{\varepsilon}\right), \quad x_{i}<\sigma
$$

hold.

Proof. Note that

$$
D^{+} V_{\varepsilon}\left(x_{i}\right)=D^{+}\left(V_{\varepsilon}-v_{\varepsilon}\right)\left(x_{i}\right)+D^{+} v_{\varepsilon}\left(x_{i}\right)-v_{\varepsilon}^{\prime}\left(x_{i}\right)+v_{\varepsilon}^{\prime}\left(x_{i}\right)
$$

and $\left\|v_{\varepsilon}^{\prime}\right\| \leqslant C$ and as in [5] $\left|D^{+} v_{\varepsilon}\left(x_{i}\right)-v_{\varepsilon}^{\prime}\left(x_{i}\right)\right| \leqslant C N^{-1}$. Hence,

$$
\left|D^{+} V_{\varepsilon}\left(x_{i}\right)\right| \leqslant\left|D^{+}\left(V_{\varepsilon}-v_{\varepsilon}\right)\left(x_{i}\right)\right|+C .
$$

On $[\sigma, 1], \quad\left|V_{\varepsilon}-v_{\varepsilon}\left(x_{i}\right)\right| \leqslant C N^{-1}$ and so

$$
\left|D^{+}\left(V_{\varepsilon}-v_{\varepsilon}\right)\left(x_{i}\right)\right| \leqslant C, \quad x_{i} \geqslant \sigma .
$$

As in [5] on $[0, \sigma), \varepsilon\left|D^{+}\left(V_{\varepsilon}-v_{\varepsilon}\right)\left(x_{i}\right)\right| \leqslant C N^{-1}$, which completes the proof.

In order to prove an $\varepsilon$-uniform convergence result we need to impose a further restriction on the data of problem (2.1). Namely, we require that

$$
\varepsilon\left\|w_{\varepsilon}^{\prime}\right\|<\frac{\alpha^{2}}{4 M}
$$

where $M$ is given in (2.1d). Since $\varepsilon\left\|w_{\varepsilon}^{\prime}\right\| \leqslant C$, this assumption restricts the permissible class of nonlinearities and also prevents the discrepancy of the boundary values, i.e., $|A-B|$, from becoming too large.

Theorem 3.2. Let $u_{\varepsilon}$ be the solution of (2.1) and $U_{\varepsilon}$ be the solution of (3.1). Assume that (3.4) is satisfied. The nonlinear finite difference method is $\varepsilon$-uniform on the fitted mesh $\bar{\Omega}_{\varepsilon}^{N}$ and the following $\varepsilon$-uniform error estimate holds

$$
\sup _{0<\varepsilon \leqslant \varepsilon_{1}}\left\|U_{\varepsilon}-u_{\varepsilon}\right\|_{\bar{\Omega}_{\varepsilon}^{N}} \leqslant C N^{-1} \ln N
$$

for sufficiently small $\varepsilon_{1}$. 
Proof. We consider first the case of $\sigma<0.5$. Note that

$$
\left\{\varepsilon \delta^{2}+b\left(U_{\varepsilon}\right) D^{+}\right\} U_{\varepsilon}=\left\{\varepsilon \delta^{2}+b\left(V_{\varepsilon}\right) D^{+}\right\} V_{\varepsilon} .
$$

Hence

$$
\begin{gathered}
\varepsilon \delta^{2} W_{\varepsilon}+\left(b\left(U_{\varepsilon}\right)-b\left(V_{\varepsilon}\right)\right) D^{+} V_{\varepsilon}+b\left(U_{\varepsilon}\right) D^{+} W_{\varepsilon}=\varepsilon \delta^{2} W_{\varepsilon}+b\left(U_{\varepsilon}\right) D^{+} W_{\varepsilon}+\left(b^{\prime}(\hat{W}) D^{+} V_{\varepsilon}\right) W_{\varepsilon}=0 \\
W_{\varepsilon}(0)=w_{\varepsilon}(0), \quad W_{\varepsilon}(1)=0 .
\end{gathered}
$$

The expression $\left|W_{\varepsilon}-w_{\varepsilon}\right|$ is estimated on $[0, \sigma]$ and $[\sigma, 1]$ separately as follows. In $[\sigma, 1]$, that is away from the layer, from Theorem 2 we have

$$
\left|w_{\varepsilon}(x)\right| \leqslant C \exp \left(-\alpha \varepsilon^{-1} \sigma\right) \leqslant C \exp (-\ln N)=C N^{-1} .
$$

Note that we require $N$ sufficiently large and $\varepsilon$ sufficiently small so that

$$
b\left(U_{\varepsilon}\right)^{2}-4 \varepsilon\left(b^{\prime}(\hat{W}) D^{+} V_{\varepsilon}\right) \geqslant \alpha^{2}-4 \varepsilon C\left(1+\frac{N^{-1}}{\varepsilon}\right)>0 .
$$

We now introduce the transformation

$$
W_{\varepsilon}=\lambda\left(x_{i}\right) \hat{W}_{\varepsilon}
$$

where $\left|\lambda\left(x_{i}\right)\right| \leqslant C$ is constructed so that, for $\varepsilon \leqslant \varepsilon_{0}$ sufficiently small and $N \geqslant N_{0}$ sufficiently large $\left(\varepsilon_{0}\right.$ independent of $N$ and $N_{0}$ independent of $\varepsilon$ )

$$
\hat{L}_{\varepsilon}^{N} W_{\varepsilon}=\left\{\varepsilon \delta^{2}+\hat{b} D^{+}-\hat{c}\right\} \hat{W}_{\varepsilon}=0, \quad \hat{b} \geqslant \alpha, \quad \hat{c} \geqslant \gamma_{0}>0
$$

and $\quad \hat{W}_{\varepsilon}(0)=\left|w_{\varepsilon}(0)\right|, \quad \hat{W}_{\varepsilon}(1)=0$. It is shown in the appendix that we can choose the barrier function, for example, as

$$
\lambda\left(x_{i}\right)=\prod_{j=1}^{i}\left(1+\zeta_{1} \varepsilon^{-1} N^{-1} h_{j}\right)^{-1}, \quad i<N / 2
$$

and

$$
\lambda\left(x_{i}\right)=\left(1+\zeta_{1} \varepsilon^{-1} N^{-1} h\right)^{-N / 2} \prod_{j=N / 2}^{i}\left(1+\zeta_{2} h_{j}\right)^{-1}, \quad i \geqslant N / 2
$$

where $h_{j}=x_{j}-x_{j-1}, h=2 \sigma / N$ and $\zeta_{2}>\zeta_{1}>0$ are suitable constants. Note that $\hat{L}_{\varepsilon}^{N}$ satisfies a discrete comparison principle. Using this, we deduce that

$$
\left|\hat{W}_{\varepsilon}\right| \leqslant \tilde{W}_{\varepsilon} \leqslant C N^{-1}
$$

where $\tilde{W}_{\varepsilon}$ is the solution of

$$
\begin{gathered}
\left\{\varepsilon \delta^{2}+\alpha D^{+}\right\} \tilde{W}_{\varepsilon}=0, \\
\tilde{W}_{\varepsilon}(0)=\left|w_{\varepsilon}(0)\right|, \quad \tilde{W}_{\varepsilon}(1)=0,
\end{gathered}
$$

Hence,

$$
\left|w_{\varepsilon}\left(x_{i}\right)-W_{\varepsilon}\left(x_{i}\right)\right| \leqslant C N^{-1}, \quad N / 2 \leqslant i \leqslant N
$$


On the other hand, in the layer region $[0, \sigma]$, we have

$$
\begin{aligned}
L_{U_{\varepsilon}}^{N}\left(w_{\varepsilon}-W_{\varepsilon}\right) & =L_{U_{\varepsilon}}^{N} w_{\varepsilon}-L_{u_{\varepsilon}} w_{\varepsilon} \\
& =\varepsilon\left(\delta^{2} w_{\varepsilon}-w_{\varepsilon}^{\prime \prime}\right)+b\left(U_{\varepsilon}\right) D^{+} w_{\varepsilon}-b\left(u_{\varepsilon}\right) w_{\varepsilon}^{\prime} \\
& =\varepsilon\left(\delta^{2} w_{\varepsilon}-w_{\varepsilon}^{\prime \prime}\right)+\left(b\left(U_{\varepsilon}\right)-b\left(u_{\varepsilon}\right)\right) w_{\varepsilon}^{\prime}+b\left(U_{\varepsilon}\right)\left(D^{+} w_{\varepsilon}-w_{\varepsilon}^{\prime}\right) \\
& =\varepsilon\left(\delta^{2} w_{\varepsilon}-w_{\varepsilon}^{\prime \prime}\right)+\left(b_{u}\left(\zeta_{i}\right)\left(U_{\varepsilon}-u_{\varepsilon}\right)\right) w_{\varepsilon}^{\prime}+b\left(U_{\varepsilon}\right)\left(D^{+} w_{\varepsilon}-w_{\varepsilon}^{\prime}\right) .
\end{aligned}
$$

We introduce the linear difference operator

$$
M_{U}^{N} Z \equiv\left(\varepsilon \delta^{2}+b\left(U_{\varepsilon}\right) D^{+}+\left(b_{u}\left(\zeta_{i}\right)\right) w_{\varepsilon}^{\prime}\right) Z,
$$

where $\zeta_{i}$ is defined implicitly by

$$
b_{u}\left(\zeta_{i}\right)\left(U_{\varepsilon}-u_{\varepsilon}\right) \equiv b\left(U_{\varepsilon}\right)-b\left(u_{\varepsilon}\right) .
$$

This finite difference operator satisfies a discrete maximum principle, provided that the following inequality holds for all $x$

$$
b^{2}\left(U_{\varepsilon}\right)-4 \varepsilon\left(b_{u}\left(\zeta_{i}\right) w_{\varepsilon}^{\prime}\right)>0,
$$

which is satisfied by virtue of assumption (3.4). Recalling the expression for the truncation error

$$
M_{U}^{N}(w-W)=\varepsilon\left(\delta^{2} w_{\varepsilon}-w_{\varepsilon}^{\prime \prime}\right)+b(U)\left(D^{+} w_{\varepsilon}-w_{\varepsilon}^{\prime}\right)+b_{u}\left(\zeta_{i}\right) w_{\varepsilon}^{\prime}\left(V_{\varepsilon}-v_{\varepsilon}\right),
$$

the argument now follows that of the linear case (see [5]), using a comparison principle and an appropriate barrier function. The case of $\sigma=0.5$ is dealt with as in the case of the fine mesh region above (see [5]).

As in the linear case (see [5]), this nodal error bound extends, for sufficiently small $\varepsilon_{1}$, to the global convergence result

$$
\sup _{0<\varepsilon \leqslant \varepsilon_{1}}\left\|\bar{U}_{\varepsilon}-u_{\varepsilon}\right\|_{\bar{\Omega}} \leqslant C N^{-1} \ln N
$$

where $\bar{U}_{\varepsilon}$ is the piecewise linear interpolant of $U_{\varepsilon}$ on $\bar{\Omega}$.

We end this section by showing that the class of problems satisfying (3.4) is not trivial. Consider the following class of quasilinear problems:

$$
\begin{gathered}
\varepsilon u_{\varepsilon}^{\prime \prime}+u_{\varepsilon}(x) u_{\varepsilon}^{\prime}=0, \quad x \in(0,1), \\
u_{\varepsilon}(0)=A, \quad u_{\varepsilon}(1)=B .
\end{gathered}
$$

From $(2.2)$ and the fact that $b\left(u_{\varepsilon}\right)=u_{\varepsilon}$ we see that

$$
\alpha=\min \{A, B\} \leqslant u_{\varepsilon}(x) \leqslant \max \{A, B\}=\beta
$$

and that we can take $M=1 \mathrm{in}(2.1 \mathrm{~d})$. Then condition (2.1c) restricts the boundary values to being strictly positive. Note that for problems of the form $(3.6)$ we have $v_{\varepsilon}(x)=B$, a constant function, and thus $w_{\varepsilon}^{\prime}=u_{\varepsilon}^{\prime}$. The assumption (3.4) on the data reduces to

$$
\alpha^{2}-4 \varepsilon\left\|u_{\varepsilon}^{\prime}\right\|>0
$$


Integrating (3.6) from 0 to $x$ yields

$$
\varepsilon u_{\varepsilon}^{\prime}(x)+\frac{1}{2} u_{\varepsilon}^{2}(x)=\varepsilon u_{\varepsilon}^{\prime}(0)+\frac{1}{2} u_{\varepsilon}^{2}(0) .
$$

Integrating this from 0 to 1 yields

$$
\begin{aligned}
\varepsilon u_{\varepsilon}^{\prime}(x) & =\varepsilon\left(u_{\varepsilon}(1)-u_{\varepsilon}(0)\right)+\frac{1}{2}\left(\int_{t=0}^{1} u_{\varepsilon}^{2}(t) d t-u_{\varepsilon}^{2}(x)\right) \\
& =\varepsilon\left(u_{\varepsilon}(1)-u_{\varepsilon}(0)\right)+\frac{1}{2}\left[u_{\varepsilon}^{2}(\hat{x})-u_{\varepsilon}^{2}(x)\right] .
\end{aligned}
$$

Hence

$$
\left|\varepsilon u_{\varepsilon}^{\prime}(x)\right| \leqslant \varepsilon|B-A|+\frac{1}{2}\left(\beta^{2}-\alpha^{2}\right)
$$

and thus assumption (3.4) becomes

$$
3 \alpha^{2}-2 \beta^{2}-4 \varepsilon(\beta-\alpha)>0 .
$$

For $\varepsilon$ sufficiently small, our assumptions become

$$
\alpha, \beta>0 \quad \text { and } 3 \alpha^{2}-2 \beta^{2}>0,
$$

where $\alpha=\min \{A, B\}$ and $\beta=\max \{A, B\}$. This is a nontrivial class of problems.

\section{Numerical results}

All of the numerical results in this section are for the one-dimensional quasilinear problem (3.6). Various choices of $A$ and $B$ in the boundary conditions are examined. We will examine the performance of two numerical methods applied to problem (3.6). The first method consists of a fitted finite difference operator (denoted by $L_{\varepsilon}^{N}$ ) combined with a uniform mesh $\bar{\Omega}_{u}^{N}=\{i h\}_{i=0}^{N}$. The second method consists of a standard upwind finite difference operator (denoted by $L_{u p}^{N}$ ) combined with a fitted piecewise-uniform mesh (denoted by $\bar{\Omega}_{\varepsilon}^{N}$ ), which was defined in the previous section.

The first method is a nonlinear extension to problem (3.6) of the fitted operator method of Il'in-Allen-Southwell. The method is

$$
\begin{gathered}
\varepsilon^{h}\left(U_{\varepsilon}(x) h / \varepsilon\right) \delta_{x}^{2} U_{\varepsilon}(x)+U_{\varepsilon}(x) D_{x}^{+} U_{\varepsilon}(x)=0, \quad x \in \Omega_{u}^{N} \\
U_{\varepsilon}(0)=u_{\varepsilon}(0), \quad U_{\varepsilon}(1)=u_{\varepsilon}(1)
\end{gathered}
$$

where $\varepsilon^{h}\left(U_{\varepsilon}(x) h / \varepsilon\right)$ is given by $\varepsilon^{h}(y)=B(y)=y /\left(e^{y}-1\right)$ which is the Bernoulli generating function. The nonlinear El-Mistikawy and Werle method for problem (3.6) is similar but more complicated.

Note that it is well known theoretically that the Il'in-Allen-Southwell and the El-Mistikawy and Werle methods are $\varepsilon$-uniform for certain linear singular perturbation problems. We show below, by numerical experiments, that the above nonlinear counterparts are not $\varepsilon$ uniform for problem (3.6). 
The second numerical method for problem (3.6) is

$$
\begin{gathered}
\varepsilon \delta_{x}^{2} U_{\varepsilon}(x)+U_{\varepsilon}(x) D_{x}^{+} U_{\varepsilon}(x)=0, \quad x \in \Omega_{\varepsilon}^{N}, \\
U_{\varepsilon}(0)=A, \quad U_{\varepsilon}(1)=B,
\end{gathered}
$$

where $\bar{\Omega}_{\varepsilon}^{N}$ is defined in Section 3 .

Both of these nonlinear finite difference methods are solved by the following continuation method, which is a generalization to fitted operator methods of the one described in [4]. The related nonlinear upwind finite difference equation in the continuation method is

$$
\left(\varepsilon^{h} \delta_{x}^{2}+U_{\varepsilon}(x, t) D_{x}^{+}-D_{t}^{-}\right) U_{\varepsilon}(x, t)=0, \quad x \in \Omega^{N}, \quad \varepsilon^{h}(x, t)=B\left(U_{\varepsilon}(x, t) h / \varepsilon\right)
$$

where $t$ is the positive time-like continuation parameter and $D_{t}^{-}$is the backward difference operator

$$
D_{t}^{-} U_{\varepsilon}\left(x, t_{j}\right)=\left(t_{j}-t_{j-1}\right)^{-1}\left(U_{\varepsilon}\left(x, t_{j}\right)-U_{\varepsilon}\left(x, t_{j-1}\right)\right) .
$$

Now consider the iterative linear finite difference method

$$
\left(\varepsilon^{h, o l d} \delta_{x}^{2}+U_{\varepsilon}^{o}(x, t) D_{x}^{+}-D_{t}^{-}\right) U_{\varepsilon}(x, t)=0, \quad(x, t) \in G^{N},
$$

where $U_{\varepsilon}^{o}(x, t)$ is the old value of $U_{\varepsilon}$ defined by $U_{\varepsilon}^{o}\left(x, t_{j}\right)=U_{\varepsilon}\left(x, t_{j-1}\right)$ and $\varepsilon^{h, o l d}(x, t)=$ $B\left(U_{\varepsilon}^{o}(x, t) h / \varepsilon\right)$. The mesh $G^{N}$ is defined by

$$
\bar{G}^{N}=\bar{\Omega}^{N} \times \bar{\omega}_{t}
$$

where $\bar{\omega}_{t}=\left\{t_{j}\right\}_{j=0}^{K}$ is a uniform mesh on $[0, T]$ such that $t_{0}=0, t_{K}=T$. The mesh $\bar{\Omega}^{N}$ is the mesh corresponding to the original nonlinear finite difference method. In the case of the fitted operator methods $\bar{\Omega}^{N}$ is the uniform mesh $\bar{\Omega}_{u}^{N}$ and in the case of the fitted mesh method $\bar{\Omega}^{N}$ is the fitted piecewise-uniform mesh $\bar{\Omega}_{\varepsilon}^{N}$ constructed in the previous section. The following boundary and initial conditions are imposed

$$
\begin{gathered}
U_{\varepsilon}(0, t)=A, \quad U_{\varepsilon}(1, t)=B, \quad t \in[0, T], \\
U_{\varepsilon}(x, 0)=u_{\text {init }}(x), \quad x \in(0,1),
\end{gathered}
$$

where $u_{\text {init }}(x)$ is a bounded function, which is an appropriate initial guess for the iteration procedure.

Since the finite difference method (4.3) is implicit, at each time level the function $U_{\varepsilon}(x, t)$ is the solution of a discrete linear boundary value problem. Note that the operator $\varepsilon^{h} \delta_{x}^{2}+$ $U_{\varepsilon}(x, t) D_{x}^{+}-D_{t}^{-}$is monotone on the mesh $G_{\varepsilon}^{N}$ for any distribution of the mesh points in $\bar{\omega}_{t}$.

In each case the nonlinear upwind finite difference operator is linearized using the above continuation method. That is,

$$
\begin{gathered}
\left(\varepsilon^{h} \delta_{x}^{2} U_{\varepsilon}-U_{\varepsilon}\left(x, t_{j-1}\right) D_{x}^{+} U_{\varepsilon}-D_{t}^{-} U_{\varepsilon}\right)\left(x, t_{j}\right)=f(x), \quad j=1, \ldots K, \\
U_{\varepsilon}\left(0, t_{j}\right)=u(0), \quad U_{\varepsilon}\left(1, t_{j}\right)=u(1) \text { for all } j, \\
U_{\varepsilon}(x, 0)=u_{\text {init }}(x) .
\end{gathered}
$$

Various $u_{\text {init }}(x)$ are chosen. The choices of the uniform time-like step $k=t_{j}-t_{j-1}$ and the number of iterations $K$ are determined as follows. Defining

$$
e(j) \equiv \max _{1 \leqslant i \leqslant N}\left|U_{\varepsilon}\left(x_{i}, t_{j}\right)-U_{\varepsilon}\left(x_{i}, t_{j-1}\right)\right| / k, \quad \text { for } j=1,2, \cdots, K
$$


the time-like step $k$ is chosen sufficiently small so that

$$
e(j) \leqslant e(j-1), \quad \text { for all } j \text { satisfying } 1<j \leqslant K \text {. }
$$

Then the number of iterations $K$ is chosen such that

$$
e(K) \leqslant \mathrm{TOL}
$$

where TOL is a suitably prescribed small tolerance.

The numerical solution is computed using the following algorithm. Start from $t_{0}$ with the initial timestep $k=1.0$. If, at some value of $j,(4.5 \mathrm{~b})$ is not satisfied, then discard the timestep from $t_{j-1}$ to $t_{j}$ and restart from $t_{j-1}$ with half the time step, that is $k^{\text {new }}=k / 2$, and continue halving the timestep until one finds a $k$ for which (4.5b) is satisfied. Assuming that $(4.5 \mathrm{~b})$ is satisfied at each timestep, continue until either $(4.5 \mathrm{c})$ is satisfied or $t_{j}=1000$. or the $t_{j}-t_{j-1}<.000001$. If $(4.5 \mathrm{c})$ is not satisfied, we assume that the timestepping process stalled due to a too large choice of the initial timestep. In this case we repeat the entire process again from $t_{0}$, halving the initial timestep $k$ to $k=0.5$. If the process stalls again, we restart from $t_{0}$ again halving the initial timestep. If $(4.5 \mathrm{c})$ is satisfied, the resulting values of $U_{\varepsilon}(x, K)$ are taken as the approximations to the solution of the continuous problem (3.6).

Each problem is solved on a sequence of meshes, with $N=8,16 \ldots 1024$ and for $\varepsilon=$ $2^{-n}, n=1,2, \cdots j_{\text {red }}$, where $j_{\text {red }}$ is chosen so that $\varepsilon=2^{-j_{\text {red }}}$ is a value at which the rate of convergence stabilizes. This normally occurs when, to machine accuracy, the reduced problem is being solved.

Since the exact solution is usually unknown, the errors $\left|U_{\varepsilon}\left(x_{i}, K\right)-u_{\varepsilon}\left(x_{i}\right)\right|$ are approximated on each mesh, for successive values of $\varepsilon$, by $e_{\varepsilon}^{N}(i)=\left|U_{\varepsilon}\left(x_{i}, K\right)-u_{\varepsilon}^{I}\left(x_{i}, K_{\varepsilon}\right)\right|$, where the interpolant $u_{\varepsilon}^{I}\left(x, K_{\varepsilon}\right)$ is defined, using linear interpolation on each subinterval $\left[y_{j-1}, y_{j}\right]$, by

$$
u_{\varepsilon}^{I}\left(x, K_{\varepsilon}\right)=U_{\varepsilon}^{*}\left(y_{j-1}, K_{\varepsilon}\right)+\left(U_{\varepsilon}^{*}\left(y_{j}, K_{\varepsilon}\right)-U_{\varepsilon}^{*}\left(y_{j-1}, K_{\varepsilon}\right)\right) \frac{x-y_{j-1}}{y_{j}-y_{j-1}}, \quad 1 \leqslant j \leqslant 1024
$$

and where the nodal values $\left\{U_{\varepsilon}^{*}\left(y_{j}, K_{\varepsilon}\right)\right\}_{j=0}^{1024}, y_{j} \in \bar{\Omega}_{\varepsilon}^{N}$ are obtained from the solution of the finite difference method (4.3) with $N=1024$. Here $\bar{\Omega}_{\varepsilon}^{N}$ and $K_{\varepsilon}$ are the fitted piecewiseuniform mesh and the related number of iterations in the stopping criteria (4.5c), respectively. For each $\varepsilon$ and each $N$ the maximum nodal error is then approximated by the computed quantities

$$
E_{\varepsilon}^{N}=\max _{i} e_{\varepsilon}^{N}(i)
$$

and, for each $N$, the $\varepsilon$-uniform maximum nodal error is approximated by the computed quantities

$$
E^{N}=\max _{\varepsilon} E_{\varepsilon}^{N}
$$

The numerical approximations $U_{\varepsilon}$ produced by the above iterative method are said to be $\varepsilon$ uniform of order $p$ on the mesh $\Omega^{N}=\left\{x_{i}, i=0,1, \ldots, N\right\}$, if there exists constants $N_{0}, K, C$ and $p>0$ independent of $\varepsilon$ and $N$ such that for all $N \geqslant N_{0}$

$$
\max _{\Omega^{N}}\left|U_{\varepsilon}(x, K)-u_{\varepsilon}(x)\right| \leqslant C N^{-p},
$$

where $u_{\varepsilon}$ is the exact solution of the problem. 
Numerical approximations of the $\varepsilon$-uniform order of convergence $p$ are now determined using the double mesh method described, for example, in [5]. This is achieved by calculating the double mesh error

$$
D_{\varepsilon}^{N}=\max _{\Omega_{N}}\left|U_{\varepsilon}^{N}\left(x_{i}, K\right)-U_{\varepsilon}^{2 N, I}\left(x_{i}, K\right)\right|,
$$

which is the difference between the values of the solution on a mesh of $N$ points and the interpolated value for the solution, at the same point, on a mesh of $2 N$ points. For each value of $N$ the quantities

$$
D^{N}=\max _{\varepsilon} D_{\varepsilon}^{N}, \quad p^{N}=\log _{2}\left(\frac{D^{N}}{D^{2 N}}\right)
$$

are then computed. The values of $p^{N}$ are taken as computed numerical approximations to $p$.

It can be shown theoretically that the Il'in-Allen-Southwell fitted operator method is not $\varepsilon$-uniform for the quasilinear problem (3.6), although it is $\varepsilon$-uniform for certain linear problems [12]. In Table 1 we present the nodal errors $E_{\varepsilon}^{N}$ and $E^{N}$ when it is applied to problem (3.6) with the boundary conditions $u_{\varepsilon}(0)=0.9, u_{\varepsilon}(1)=1.0$ and the initial guess $u_{\text {init }}(x)=u(0)+(u(1)-u(0)) x$. Since the values of $E^{N}$ in the last row of the table are not decreasing with increasing $N$, it is clear that the method is not $\varepsilon$ - uniform for this problem. There is always a value of $\varepsilon$ for which the corresponding error is of magnitude about 0.0003 regardless of how large $N$ is taken. A summary of the computed errors $E^{N}$ and the computed $\varepsilon$-uniform orders of convergence $p^{N}$ for the method is presented in Table 2.

Table 1. Computed errors $E_{\varepsilon}^{N}$ and $E^{N}$ for the Il'in-Allen-Southwell method applied to the quasilinear

\begin{tabular}{|c|c|c|c|c|c|c|}
\hline \multicolumn{7}{|c|}{ Boundary Conditions: $\quad u(0)=0.9, \quad u(1)=1.0$} \\
\hline \multicolumn{3}{|c|}{ Initial Guess : } & \multicolumn{4}{|c|}{$u_{\text {init }}(x)=u(0)+(u(1)-u(0)) x$} \\
\hline \multicolumn{7}{|c|}{ Number of Mesh Points $N$} \\
\hline$\varepsilon$ & 8 & 16 & 32 & 64 & 128 & 256 \\
\hline $2^{-1}$ & .000012 & .000003 & .000001 & .000000 & .000000 & .000000 \\
\hline $2^{-2}$ & .000047 & .000013 & .000003 & .000001 & .000000 & .000000 \\
\hline $2^{-3}$ & .000176 & .000047 & .000013 & .000003 & .000001 & .000000 \\
\hline $2^{-4}$ & .000277 & .000176 & .000047 & .000013 & .000003 & .000001 \\
\hline $2^{-5}$ & .000081 & .000277 & .000176 & .000047 & .000012 & .000003 \\
\hline $2^{-6}$ & .000002 & .000081 & .000277 & .000175 & .000046 & .000012 \\
\hline $2^{-7}$ & .000000 & .000002 & .000081 & .000276 & .000173 & .000044 \\
\hline $2^{-8}$ & .000000 & .000000 & .000002 & .000080 & .000271 & .000164 \\
\hline $2^{-9}$ & .000000 & .000000 & .000000 & .000002 & .000077 & .000253 \\
\hline $2^{-10}$ & .000000 & .000000 & .000000 & .000000 & .000001 & .000067 \\
\hline $2^{-11}$ & .000000 & .000000 & .000000 & .000000 & .000000 & .000001 \\
\hline $2^{-12}$ & .000000 & .000000 & .000000 & .000000 & .000000 & .000000 \\
\hline $2^{-18}$ & .000000 & .000000 & .000000 & . 000000 & .000000 & .000000 \\
\hline$E^{N}$ & .000277 & .000277 & .000277 & .000276 & .000271 & .000253 \\
\hline
\end{tabular}
problem (3.6) 
Table 2. Computed errors $E^{N}$ and $\varepsilon$-uniform orders of convergence $p^{N}$ for the Il'in-Allen-Southwell method applied to the quasilinear problem (3.6).

\begin{tabular}{|c|c|c|c|c|c|c|}
\hline \multicolumn{7}{|c|}{ Boundary Conditions: $\quad u(0)=0.9, \quad u(1)=1.0$} \\
\hline \multicolumn{3}{|c|}{ Initial Guess : } & \multicolumn{4}{|c|}{$u_{\text {init }}(x)=u(0)+(u(1)-u(0)) x$} \\
\hline$N$ & 8 & 16 & 32 & 64 & 128 & 256 \\
\hline$E^{N}$ & .000277 & 等277 & .000277 & .000276 & .000271 & .000253 \\
\hline$p^{N}$ & 0.00 & 0.00 & 0.00 & 0.00 & 0.00 & 0.00 \\
\hline
\end{tabular}

In Table 3 a similar summary is given for the El-Mistakawy and Werle method [2], which is known to be $\varepsilon$-uniform of order $h^{2}$ for certain linear problems [16]. Since the values of $p^{N}$ decrease as $N$ increases, it is clear that the method is not $\varepsilon$-uniform for this quasilinear problem. In summary the numerical results in Tables 1-3 indicate clearly that on a uniform

Table 3. Computed errors $E^{N}$ and $\varepsilon$-uniform orders of convergence $p^{N}$ for the El-Mistikawy and Werle method applied to the quasilinear problem (3.6).

\begin{tabular}{|c|c|c|c|c|c|c|}
\hline \multicolumn{7}{|c|}{ Boundary Conditions: $u(0)=0.9, \quad u(1)=1.0$} \\
\hline \multicolumn{3}{|c|}{ Initial Guess : } & \multicolumn{4}{|c|}{$u_{\text {init }}(x)=u(0)+(u(1)-u(0)) x$} \\
\hline$N$ & 8 & 16 & 32 & 64 & 128 & 256 \\
\hline$E^{N}$ & .001753 & .001069 & 0723 & .000549 & .000457 & .000393 \\
\hline$p^{N}$ & .72 & .57 & .41 & .26 & .15 & .08 \\
\hline
\end{tabular}

mesh two of the best known fitted operator methods are not $\varepsilon$-uniform for a simple quasilinear problem, even though they are $\varepsilon$-uniform for some linear problems.

We now apply the $\varepsilon$-uniform fitted mesh method developed above to problem (3.6). In this case the continuation method is simpler than the fitted operator method since $\varepsilon^{h}(x, t) \equiv \varepsilon$.

In Table 4 we display the computed errors $E_{\varepsilon}^{N}$ and $E^{N}$ for problem (3.6) with the boundary conditions $u_{\varepsilon}(0)=0.9, u_{\varepsilon}(1)=1.0$ and the initial guess $u_{\text {init }}(x)=u_{\varepsilon}(0)+\left(u_{\varepsilon}(1)-u_{\varepsilon}(0)\right) x$ obtained using the fitted piecewise-uniform mesh $\bar{\Omega}_{\varepsilon}^{N}$ for various values of $\varepsilon$ and $N$. In Table 5 the number of iterations required for the continuation method to converge are given. In the case of these iteration counts, there was no halving of the time-step and no restarts. Therefore, the number of iterations is the number of steps. Since the initial time-step is taken to be 1.0, the number of iterations is also the time at which convergence is achieved. The number of iterations stabilizes for small $\varepsilon$ and hence is uniform in $\varepsilon$. The number of iterations appears not to increase with $N$.

In Table 6 we present summary results for problem (3.6) using the same method for various choices of initial guess and a different set of boundary conditions.

It is clear from these tables that the $\varepsilon$-uniform error and $\varepsilon$-uniform order of convergence are independent of the initial guess. Note that the $\varepsilon$-uniform error and $\varepsilon$-uniform order of convergence depend on the boundary conditions. These results validate computationally the $\varepsilon$-uniform behaviour established theoretically in Theorem 4 . 
Table 4. Computed errors $E_{\varepsilon}^{N}$ and $E^{N}$ using the upwind difference operator on the fitted mesh $\bar{\Omega}_{\varepsilon}^{N}$ for problem (3.6).

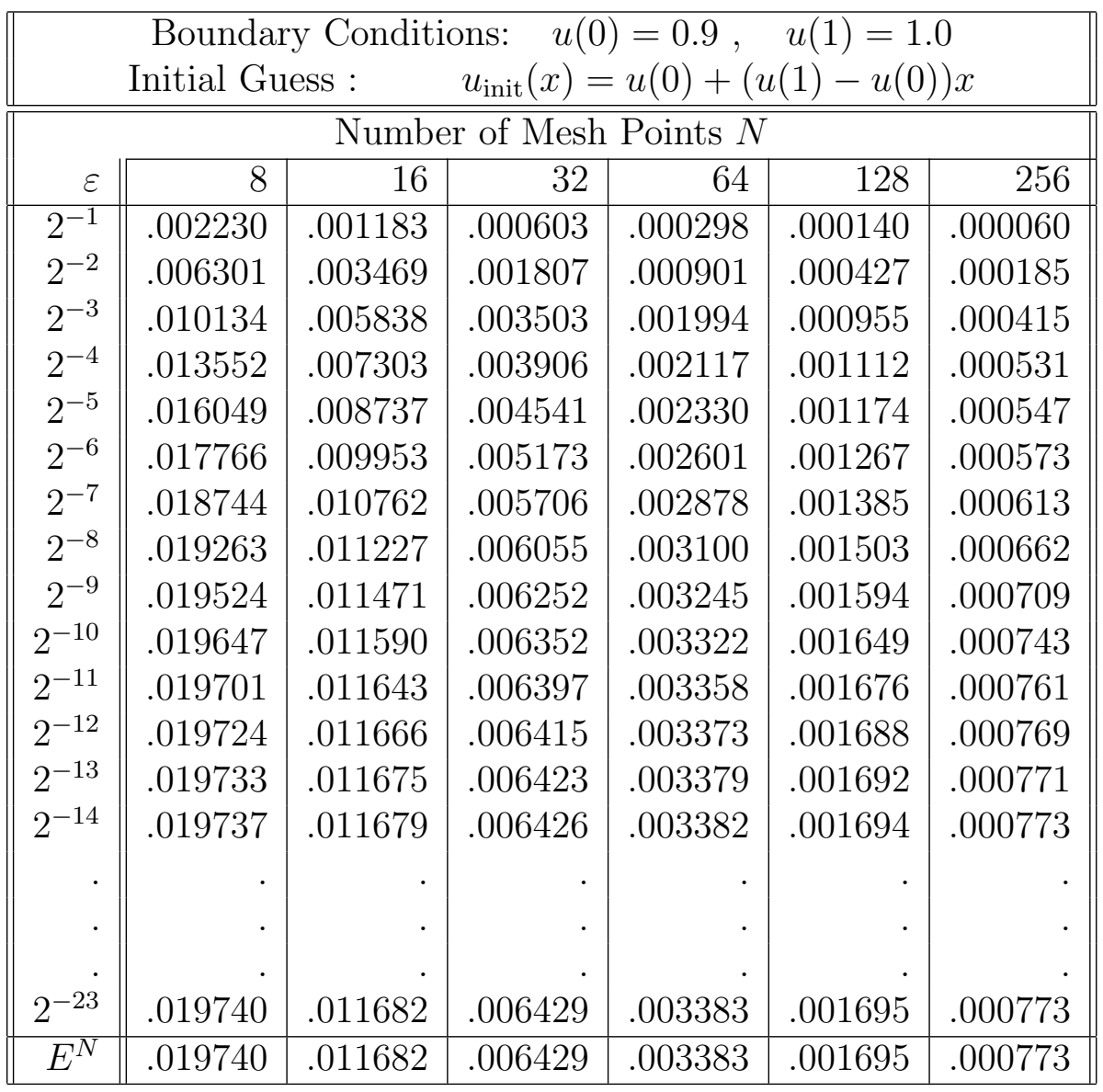

Table 5. Number of iterations using the upwind difference operator on the fitted mesh $\bar{\Omega}_{\varepsilon}^{N}$ for problem (3.6).

\begin{tabular}{|r||r|r|r|r|r|r|r||}
\hline \multicolumn{1}{|c||}{} & \multicolumn{6}{|c||}{ Number of Mesh Points $N$} \\
\cline { 2 - 8 }$\varepsilon$ & 8 & 16 & 32 & 64 & 128 & 256 & 512 \\
\hline $2^{-1}$ & 8 & 8 & 8 & 8 & 8 & 8 & 8 \\
$2^{-2}$ & 10 & 10 & 10 & 10 & 10 & 10 & 10 \\
$2^{-3}$ & 12 & 11 & 11 & 11 & 11 & 11 & 11 \\
$2^{-4}$ & 12 & 12 & 11 & 11 & 11 & 11 & 11 \\
$2^{-5}$ & 12 & 11 & 11 & 10 & 10 & 10 & 10 \\
$2^{-6}$ & 12 & 11 & 11 & 10 & 10 & 10 & 10 \\
$2^{-7}$ & 12 & 11 & 10 & 10 & 10 & 10 & 10 \\
$2^{-8}$ & 12 & 11 & 10 & 10 & 10 & 10 & 10 \\
$\cdot$ & $\cdot$ &. &. &. &. &. &. \\
$\cdot$ & $\cdot$ &. &. &. &. &. &. \\
. &. &. &. &. &. &. &. \\
$2^{-23}$ & 12 & 11 & 10 & 10 & 10 & 10 & 10 \\
\hline
\end{tabular}


Table 6. Computed errors $E^{N}$ and $\varepsilon$-uniform orders of convergence $p^{N}$ for the upwind difference operator on the fitted mesh $\bar{\Omega}_{\varepsilon}^{N}$ for problem (3.6) for various initial guesses and boundary values

\begin{tabular}{|c|c|c|c|c|c|c|}
\hline \multicolumn{7}{|c|}{$\begin{array}{cc}\text { Boundary Conditions: } & u(0)=0.5, \quad u(1)=1.5 \\
\text { Initial Guess : } & u_{\text {init }}(x)=u(0)\end{array}$} \\
\hline$N$ & 8 & 16 & 32 & 64 & 128 & 256 \\
\hline$E^{N}$ & 0.214942 & 0.110771 & 0.058319 & 0.030843 & 0.015944 & 0.007514 \\
\hline$p^{N}$ & 1.01 & 0.97 & 0.91 & 0.81 & 0.81 & 0.82 \\
\hline \multicolumn{7}{|c|}{$\begin{array}{cc}\text { Boundary Conditions: } & u(0)=0.5, \quad u(1)=1.5 \\
\text { Initial Guess : } & u_{\text {init }}(x)=u(1)\end{array}$} \\
\hline$N$ & 8 & 16 & 32 & 64 & 128 & 256 \\
\hline$E^{N}$ & 0.213530 & 0.110426 & 0.058188 & 0.030836 & 0.015957 & 0.007525 \\
\hline$p^{N}$ & 1.00 & 0.97 & 0.90 & 0.81 & 0.80 & 0.81 \\
\hline \multicolumn{7}{|c|}{ Boundary Conditions: $u(0)=0.5, \quad u(1)=1.5$} \\
\hline \multicolumn{7}{|c|}{ Initial Guess : $\quad u_{\text {init }}(x)=u(0)+(u(1)-u(0))(x+$} \\
\hline$N$ & 8 & 16 & 32 & 64 & 128 & 256 \\
\hline$E^{N}$ & 0.215027 & 0.110790 & 0.058318 & 0.030845 & 0.015946 & 0.007516 \\
\hline$p^{N}$ & 1.01 & 0.97 & 0.91 & 0.81 & 0.81 & 0.82 \\
\hline \multicolumn{7}{|c|}{ Boundary Conditions: $u(0)=0.5, \quad u(1)=1.0$} \\
\hline \multicolumn{7}{|c|}{ Initial Guess : $\quad u_{\text {init }}(x)=u(0)+(u(1)-u(0))(x+$} \\
\hline$N$ & 8 & 16 & 32 & 64 & 128 & 256 \\
\hline$E^{N}$ & 0.127947 & 0.075625 & 0.040361 & 0.020416 & 0.009910 & 0.004415 \\
\hline$p^{N}$ & 0.55 & 0.83 & 0.93 & 0.94 & 0.93 & 0.92 \\
\hline
\end{tabular}

\section{Conclusions}

A finite difference method which is $\varepsilon$-uniform in the maximum norm is constructed for a class of problems with singularly perturbed quasilinear differential equations. The numerical method comprises a standard upwind finite difference operator on fitted piecewise-uniform meshes condensing in the boundary layers. On the other hand, fitted operator methods on uniform meshes are not $\varepsilon$-uniform in the maximum norm for these problems. Numerical results supporting the above conclusions are presented.

\section{Appendix}

Recall from the proof of Theorem 4 that $W_{\varepsilon}$ is the solution of

$$
L_{\varepsilon}^{N} W_{\varepsilon}=\varepsilon \delta^{2} W_{\varepsilon}+b D^{+} W_{\varepsilon}+c W_{\varepsilon}=0, \quad W_{\varepsilon}(0)=w_{\varepsilon}(0), \quad W_{\varepsilon}(1)=0
$$

where $b=b\left(U_{\varepsilon}\right)>0$ and $c=\left(b^{\prime}(\hat{W}) D^{+} V_{\varepsilon}\right)$. In this appendix, we explicitly determine a transformation

$$
W_{\varepsilon}=\lambda\left(x_{i}\right) \hat{W}_{\varepsilon}
$$

where $\left|\lambda\left(x_{i}\right)\right| \leqslant C$ is constructed so that, for $\varepsilon \leqslant \varepsilon_{0}$ sufficiently small and $N \geqslant N_{0}$ sufficiently large ( $\varepsilon_{0}$ independent of $N$ and $N_{0}$ independent of $\varepsilon$ ),

$$
L_{\varepsilon}^{N}\left(\lambda \hat{W}_{\varepsilon}\right)=\hat{L}_{\varepsilon}^{N} \hat{W}_{\varepsilon}=\left\{\varepsilon \delta^{2}+\hat{b} D^{+}-\hat{c}\right\} \hat{W}_{\varepsilon}=0, \quad \hat{b} \geqslant \alpha, \quad \hat{c} \geqslant \gamma_{0}>0
$$


and $\hat{W}_{\varepsilon}(0)=\left|W_{\varepsilon}(0)\right|, \quad \hat{W}_{\varepsilon}(1)=0$. For any choice of the barrier function $\lambda\left(x_{i}\right)$

$$
\begin{aligned}
D^{+}\left(\lambda\left(x_{i}\right) \hat{W}_{\varepsilon}\left(x_{i}\right)\right) & =\lambda\left(x_{i+1}\right) D^{+} \hat{W}_{\varepsilon}\left(x_{i}\right)+\hat{W}_{\varepsilon}\left(x_{i}\right) D^{+} \lambda\left(x_{i}\right), \\
D^{-}\left(\lambda\left(x_{i}\right) \hat{W}_{\varepsilon}\left(x_{i}\right)\right) & =\lambda\left(x_{i-1}\right) D^{-} \hat{W}_{\varepsilon}\left(x_{i}\right)+\hat{W}_{\varepsilon}\left(x_{i}\right) D^{-} \lambda\left(x_{i}\right), \\
\delta^{2}\left(\lambda\left(x_{i}\right) \hat{W}_{\varepsilon}\left(x_{i}\right)\right) & =\hat{W}_{\varepsilon}\left(x_{i}\right) \delta^{2}\left(\lambda\left(x_{i}\right)\right)+\frac{1}{\bar{h}_{i}}\left(\lambda\left(x_{i+1}\right) D^{+} \hat{W}_{\varepsilon}\left(x_{i}\right)-\lambda\left(x_{i-1}\right) D^{-} \hat{W}_{\varepsilon}\left(x_{i}\right)\right),
\end{aligned}
$$

where $\bar{h}_{i}=\left(h_{i}+h_{i+1}\right) / 2$. Hence

$\varepsilon \delta^{2}\left(\lambda\left(x_{i}\right) \hat{W}_{\varepsilon}\left(x_{i}\right)\right)=\varepsilon \hat{W}_{\varepsilon}\left(x_{i}\right) \delta^{2}\left(\lambda\left(x_{i}\right)\right)+\frac{\varepsilon}{\bar{h}_{i}}\left(\lambda\left(x_{i+1}\right)-\lambda\left(x_{i-1}\right)\right) D^{+} \hat{W}_{\varepsilon}\left(x_{i}\right)+\varepsilon \lambda\left(x_{i-1}\right) \delta^{2}\left(\hat{W}_{\varepsilon}\left(x_{i}\right)\right)$.

Then

$$
\begin{aligned}
0= & \varepsilon \delta^{2}\left(\lambda\left(x_{i}\right) \hat{W}_{\varepsilon}\left(x_{i}\right)\right)+b D^{+}\left(\lambda\left(x_{i}\right) \hat{W}_{\varepsilon}\left(x_{i}\right)\right)+c\left(\lambda\left(x_{i}\right) \hat{W}_{\varepsilon}\left(x_{i}\right)\right)= \\
= & \lambda\left(x_{i-1}\right)\left[\varepsilon \delta^{2}\left(\hat{W}_{\varepsilon}\left(x_{i}\right)\right)+\frac{\varepsilon \delta^{2}\left(\lambda\left(x_{i}\right)\right)+b D^{+}\left(\lambda\left(x_{i}\right)\right)+c \lambda\left(x_{i}\right)}{\lambda\left(x_{i-1}\right)} \hat{W}_{\varepsilon}\left(x_{i}\right)\right. \\
& \left.+\frac{\varepsilon}{\bar{h}_{i}}\left(\left(1+\frac{b \bar{h}_{i}}{\varepsilon}\right) \frac{\lambda\left(x_{i+1}\right)}{\lambda\left(x_{i-1}\right)}-1\right) D^{+} \hat{W}_{\varepsilon}\left(x_{i}\right)\right] .
\end{aligned}
$$

Thus, in general,

$$
\hat{b}=\frac{\varepsilon}{\bar{h}_{i}}\left(\left(1+\frac{b \bar{h}_{i}}{\varepsilon}\right) \frac{\lambda\left(x_{i+1}\right)}{\lambda\left(x_{i-1}\right)}-1\right)
$$

and

$$
\hat{c}=-\frac{\varepsilon \delta^{2}\left(\lambda\left(x_{i}\right)\right)+b D^{+}\left(\lambda\left(x_{i}\right)\right)+c \lambda\left(x_{i}\right)}{\lambda\left(x_{i-1}\right)} .
$$

For the proof of Theorem 4, Lemma 2 yields the bounds

$$
\left|c\left(x_{i}\right)\right| \leqslant C, \quad x_{i} \geqslant \sigma, \quad\left|c\left(x_{i}\right)\right| \leqslant C\left(1+\varepsilon^{-1} N^{-1}\right), \quad x_{i}<\sigma .
$$

If $\varepsilon N \geqslant 1$, then the above barrier function suffices. If $\varepsilon N \leqslant 1$, we choose the barrier function as

$$
\lambda\left(x_{i}\right)=\prod_{j=1}^{i}\left(1+\zeta_{1} \varepsilon^{-1} N^{-1} h_{j}\right)^{-1}, \quad i<N / 2
$$

and

$$
\lambda\left(x_{i}\right)=\left(1+\zeta_{1} \varepsilon^{-1} N^{-1} h\right)^{-N / 2} \prod_{j=N / 2}^{i-N / 2}\left(1+\zeta_{2} h_{j}\right)^{-1}, \quad i \geqslant N / 2
$$

where $h_{j}=x_{j}-x_{j-1}, \quad h=2 \sigma / N$ and $\zeta_{2}>\zeta_{1}>0$ are suitably chosen constants.

For $i<N / 2$, we have that $\zeta_{1} \varepsilon^{-1} N^{-1} h=O\left(N^{-2} \ln N\right)$ and so for $N$ sufficiently large

$$
\hat{b} \geqslant\left(1-\theta_{1}\right)\left[b-2 N^{-1} \zeta_{1}-\varepsilon\left(\zeta_{1} \varepsilon^{-1} N^{-1}\right)^{2} h\right] \geqslant \alpha
$$


and for $\zeta_{1}$ sufficiently large

$$
\begin{aligned}
\hat{c} & \geqslant\left(1-\theta_{1}\right)\left[\zeta_{1} \varepsilon^{-1} N^{-1} b-c\left(1+\zeta_{1} \varepsilon^{-1} N^{-1} h\right)-\varepsilon\left(\zeta_{1} \varepsilon^{-1} N^{-1}\right)^{2}\right] \\
& =\left(1-\theta_{1}\right)\left[\zeta_{1} \varepsilon^{-1} N^{-1}\left(b-\zeta_{1} N^{-1}\right)-c\left(1+\zeta_{1} \varepsilon^{-1} N^{-1} h\right)\right]>0 .
\end{aligned}
$$

For $i>N / 2$, with $\varepsilon$ sufficiently small,

$$
\hat{b} \geqslant\left(1-\theta_{2}\right)\left[b-2 \varepsilon \zeta_{2}-\varepsilon\left(\zeta_{2}\right)^{2} H\right] \geqslant \alpha, \quad H=\frac{2(1-\sigma)}{N},
$$

and for $\zeta_{2}$ sufficiently large and $\varepsilon$ sufficiently small

$$
\hat{c} \geqslant\left(1-\theta_{2}\right)\left[\zeta_{2} b-c\left(1+\zeta_{2} H\right)-\varepsilon\left(\zeta_{2}\right)^{2}\right]>0 .
$$

Now we have to deal with the transition point. Then

$$
D^{+} \lambda(\sigma)=-\frac{\zeta_{2}}{1+\zeta_{2} H} \lambda(\sigma) \text { and } \quad D^{-} \lambda(\sigma)=-\zeta_{1} \varepsilon^{-1} N^{-1} \lambda(\sigma) .
$$

From these,

$$
\delta^{2} \lambda\left(x_{i}\right)=N \lambda(\sigma)\left(\zeta_{1} \varepsilon^{-1} N^{-1}-\frac{\zeta_{2}}{1+\zeta_{2} H}\right) .
$$

Then

$$
\hat{b}=\frac{1}{\left(1+\zeta_{1} \varepsilon^{-1} N^{-1} h\right)\left(1+\zeta_{2} H\right)}\left[b-\left(h \zeta_{1}+\varepsilon H N \zeta_{2}+\zeta_{1} \zeta_{2} h H\right)\right] \geqslant \alpha
$$

and to finish we choose $\zeta_{2}$ such that $\alpha \zeta_{2}>2\left(\zeta_{1}+c\right)$ in order that

$$
\hat{c}=\frac{b \zeta_{2}}{1+\zeta_{2} H}-\zeta_{1}-c+\frac{\varepsilon N \zeta_{2}}{1+\zeta_{2} H}>0 .
$$

\section{References}

[1] E. P. Doolan, J. J. H. Miller, and W. H. A. Schilders, Uniform Numerical Methods for Problems with Initial and Boundary Layers, Boole Press, Dublin, 1980.

[2] T. M. El-Mistikawy and M. J. Werle, Numerical method for boundary layers with blowing - the exponential box scheme, AIAA J., 16 (1978), pp. 749-751.

[3] P. A. Farrell, J. J. H. Miller, E. O'Riordan, and G. I. Shishkin, On the non-existence of $\varepsilon$-uniform finite difference methods on uniform meshes for semilinear two-point boundary value problems, Math. Comp., 67 (1998), No. 222, pp. 603-617.

[4] P. A. Farrell, J. J. H. Miller, E. O'Riordan, and G. I. Shishkin, A uniformly convergent finite difference scheme for a singularly perturbed semilinear equation, SIAM J. Numer. Anal., 33 (1996), No. 3, pp. $1135-1149$.

[5] P. A. Farrell, A. F. Hegarty, J. J. H. Miller, E. O'Riordan, and G. I. Shishkin, Robust Computational Techniques for Boundary Layers, Chapman and Hall/CRC Rress, Boca Raton, FL, 2000.

[6] N. Kopteva and T. Linß, Uniform second-order pointwise convergence of a central difference approximation for a quasilinear convection-diffusion problem, Preprint MATH-NM-13-99, Institut für Numerische Mathematik, TU Dresden, December 1999.

[7] V. D. Liseikin, On the numerical solution of second order equations with a small parameter affecting the highest derivatives, Chisl. Metody Mekhaniki Splosh. Sredy, Novosibirsk, 14 (1983), No. 3, pp. 98-108. 
[8] T. Linß, H.-G. Roos, and R. Vulanović, Uniform pointwise convergence on Shishkin-type meshes for quasilinear convection-diffusion problems, SIAM J. Numer. Anal., 38 (2000), No. 3, pp. 897-912.

[9] J. Lorenz, Nonlinear boundary values problems with turning points and properties of difference schemes, in: Theory and Applications of Singular Perturbations (Oberwolfach, 1981) (W. Eckhaus and E. M. de Jager, eds.), vol. 942 of Lecture Notes in Math., Springer, Berlin-New York, 1982, pp. 150-169.

[10] J. Lorenz, Nonlinear singular perturbation problems and the Engquist-Osher difference scheme, Report 8115, University of Nijmegen, 1981.

[11] P. A. Markowich, C. A. Ringhofer, S. Selberherr, and M. Lentini, A singular perturbation approach for the analysis of the fundamental semiconductor equations, IEEE Trans. Electron Devices, 30 (1983), No. 9, pp. 1165-1180.

[12] J. J. H. Miller, G. I. Shishkin, On the construction of uniformly convergent finite difference schemes for singularly perturbed problems for a quasilinear equation, in: Computational Methods for Boundary and Interior Layers in Several Dimensions (J. J. H. Miller, ed.) Boole Press, Dublin, 1991, pp. $103-118$.

[13] J. J. H. Miller, E. O'Riordan, and G. I. Shishkin, Fitted Numerical Methods for Singular Perturbion Problems. Error Estimates in the Maximum Norm for Linear Problems in One and Two Dimensions, World Scientific, Singapore, 1996.

[14] K. Niijima, On a difference scheme of exponential type for a nonlinear singular perturbation problem, Numer. Math., 46 (1985), No. 4, pp. 521-539.

[15] K. Niijima, A uniformly convergent difference scheme for a semilinear singular perturbation problem, Numer. Math., 43 (1984), No. 2, pp. 175-198.

[16] E. O'Riordan and M. Stynes, Analysis of difference schemes for singularly perturbed differential equations using a discretized Green's function, BAIL IV (Novosibirsk, 1986), Boole Press, 1986, pp. 157-168.

[17] H.-G. Roos, M. Stynes, and L. Tobiska, Numerical Methods for Singularly Perturbed Differential Equations. Convection-Diffusion and Flow Problems, Springer-Verlag, Berlin, 1996.

[18] W. V. van Roosbroeck, Theory of flows of electrons and holes in germanium and other semiconductors, Bell Syst. Tech. J., 29 (1950), pp. 560-607.

[19] G. I. Shishkin, Approximation of solutions of singularly perturbed boundary value problems with corner boundary layer, Comput. Math. Math. Phys., 27 (1987), No. 5 pp. 54-63.

[20] G. I. Shishkin, Difference scheme for solving elliptic equations with small parameters affecting the derivatives, in: Mathematical Models and Numerical Methods (Papers, Fifth Semester, Stefan Banach Internat. Math. Center, Warsaw, 1975), Banach Center Publ., No. 3, PWN, Warsaw, 1978, pp. 89-92.

[21] G. I. Shishkin, A difference approximation of a singularly perturbed quasilinear elliptic equation degenerating into a first order equation, Soviet Math. Dokl., 43 (1991), No. 2, pp. 562-566.

[22] G. I. Shishkin, A difference approximation of a singularly perturbed boundary-value problem for quasilinear elliptic equations degenerating into first order equations, Comput. Math. Math. Phys., 32 (1992), No. 4, pp. $467-480$.

[23] M. Stynes, An adaptive uniformly convergent numerical method for a semilinear singular perturbation problem, SIAM J. Numer. Anal, 26 (1989), No. 2, pp. 442-455.

[24] V. W. Weekman, Jr., and R. L. Gorring, Influence of volume change on gas-phase reactions in porous catalysts, J. Catalysis, 4 (1965), pp. 260-270. 